\title{
A Two Node Tandem Communication Network with Feedback Having DBA and NHP Arrivals
}

\author{
Ch. V. Raghavendran ${ }^{1 *}$, G. Naga Satish ${ }^{1}$, M. V. Rama Sundari², P. Suresh Varma ${ }^{3}$ \\ ${ }^{1}$ Adikavi Nannaya University, Rajahmundry, Andhra Pradesh, India. \\ 2 Department of IT, GIET, Rajahmundry, Andhra Pradesh, India. \\ ${ }^{3}$ Dept. of Computer Science, Adikavi Nannaya University, Rajahmundry, Andhra Pradesh, India. \\ * Corresponding author. Email: raghuchv@yahoo.com \\ Manuscript submitted July 31, 2014; accepted October 8, 2014. \\ doi: 10.17706/ijcee.2014.v6.861
}

\begin{abstract}
The Communication Network model studied in this paper consists of two nodes connected in tandem with feedback for both the nodes. Each node has buffer and a transmitter for holding packets and for transmitting packets respectively. The packets after transmitted by the nodes may move to the next node or returned back to the same node for retransmission in feedback. It is considered that the arrival of packets at the nodes follows Non Homogeneous Poisson (NHP) process and transmission is characterized by Poisson process. Transmission rate of both nodes are adjusted before transmission by using Dynamic Bandwidth Allocation (DBA) policy. The model is evaluated using the difference-differential equations and a probability generating function of the number of packets in the buffer connected to the transmitter. Through mathematical modeling, performance measures including average number of packets in each buffer, the probability of emptiness of the network, the average waiting time in the buffer and in the network, throughput of the transmitters, utilization and the variance of the number of packets in the buffer are derived under transient conditions.
\end{abstract}

Keywords: Feedback, tandem network, Non Homogeneous Poisson process, dynamic bandwidth allocation, performance measures.

\section{Introduction}

Queuing network models have been widely used in design, capacity planning, call centers, emergency services, manufacturing systems, etc. for performance evaluation and optimization of communication networks. Queuing networks models with finite capacity queues and feedback have been introduced and applied as more realistic models of systems with finite capacity resources and with population constrains [1]-[5]. In an open queuing network, packets enter and depart from the network. Whereas, in a closed queuing network, packets neither enter nor depart from the network. Open queuing networks can be further divided as open feed forward queuing networks and open feedback queuing networks. In an open feed forward queuing network, a packet cannot appear in the same queue for more than one time. In an open feedback queuing network, after a packet is served by a node, it may reenter the buffer of the same node.

Feedback queues play an important role in real-life service systems, where tasks may require repeated services. Tandem queues with feedback have been widely studied in the literature. Communication networks are an important application of such systems. In their article [6], Tang and Zhao, assumed Markovian arrival and service times for feedback queues. On completion of a service at the second station, 
the task either leaves the system with probability p, or goes back, along with all tasks currently waiting in the second queue, to the first station with probability $1-\mathrm{p}$. They developed a method to study properties of exactly geometric tail asymptotic as the number of tasks in the other queue increases to infinity. Tandem queues with feedback have comprehensively studied in the literature [7]-[9]. Tandem queues with feedback are more complex objects for research than tandem queues without feedback. This assertion is confirmed to some extent, e.g. by the fact that the results for tandem with feedback are presented in handbook [10] only for the case of stationary Poisson arrival process and exponential service time distribution while the systems without feedback are investigated under more general assumptions.

In general, a realistic and high speed transmission of data/voice over the network is a major issue in communication networks. With the rapid development in the recent years, variety of communication networks are designed and analyzed with effective switching techniques. Switching techniques in communication networks are - message switching, circuit switching and packet switching. The Packet switching gives better utilization than the remaining two. In this switching technique, the message is divided into small packets of random size. The delay in packet switching can be reduced by utilizing the statistical multiplexing in communication network. The statistical multiplexing utilizes the channel capacity of the network efficiently. According to [11], [12], networks that support tele-processing applications are mixed with dynamic engineering skills and statistical multiplexing. A communication network is analyzed by considering the problems of flow control, routing, bit-dropping, delays, allocation and distribution of data/voice packetization etc., for efficient utilization of the resources. Varying the transmission rates based on the size of the buffer is to be considered for efficient transmission with high quality of service.

Adjusting the transmission rates based on the content of the buffer is known as Dynamic Bandwidth Allocation (DBA) [13]. This strategy has grown as an alternate for bit dropping and flow control strategies for quality in transmission and to reduce the congestion in buffers [14]-[17]. The strategy of dynamic bandwidth allocation is to utilize a large portion of the unutilized bandwidth. In the literature we found some works concerning communication networks with DBA. In [16], P. Suresh Varma et al. have studied the communication network model with an assumption that the transmission rate of packet is adjusted instantaneously depending upon the content of the buffer. In this they have assumed the arrival of the packets follow a Poisson process. In [18], [19], Rama Sundari et al. have developed and analyzed a three node communication network model with Non Homogeneous Poisson arrivals. It is further assumed that transmission time required by each packet at each node is dependent on the content of the buffer connected to it. Ch V. Raghavendran et al. [20]-[22] have developed two node tandem communication network model with dynamic bandwidth allocation and feedback for the first node and second node. They have presented the sensitivity analysis of the models with varying values for the parameters. In [21] they have compared the performance of the developed communication network for homogeneous and nonhomogeneous Poisson arrivals. Generally, conducting laboratory experiments with varying load conditions of a communication system in particular with DBA is difficult and complicated. Hence, mathematical models of communication networks are developed to evaluate the performance of the newly proposed communication network model under transient conditions.

In the above mentioned papers, the packets are transmitted from one node to another node in tandem or the packets may leave the network. But, practically in communication networks the packets after transmitted from a node may move to the next node or returned back to the same node for retransmission. So, in this paper we have developed and analyzed a two node communication network model having nonhomogeneous Poisson arrival and dynamic bandwidth allocation with feedback for both nodes. Here it is assumed that the packets arrive at the first node directly with time dependent arrival rate. After getting transmitted from the first transmitter the packets may join the second node connected in tandem with first 
node or returned back to the first buffer for retransmission with certain probability. Similarly, the packets transmitted by the second transmitter may leave the network or returned back to the second buffer for retransmission with certain probability. Using difference-differential equations the transient behavior of the model is analyzed by deriving the joint probability generating function of the number of packets in each buffer. The performance measures like average number of packets in the buffer and in the network; the average waiting time of packets in the buffer and in the network, throughput of the transmitter etc., of the developed network model are derived explicitly. This model is useful for evaluating communication networks where arrivals are time dependent.

\section{Network Model with DBA and NHP Arrivals with Feedback}

We consider an open queuing model of tandem communication network with two nodes. Each node consists of a buffer and a transmitter. The two buffers are $Q_{1}, Q_{2}$ and transmitters are $S_{1}, S_{2}$ connected in tandem. The arrival of packets at the first node follows non homogeneous Poisson processes with a mean arrival rate as a function of $t$ and is in the form of $\lambda(t)=\lambda+\alpha t$. It is also assumed that the packets are transmitted through the transmitters and the mean service rate in the transmitter is linearly dependent on the content of the buffer connected to it. It is assumed that the packet after getting transmitted through first transmitter may join the second buffer which is in series connected to $S_{2}$ or may be returned back buffer connected to $S_{1}$ for retransmission with certain probabilities. The packets delivered from the first node and arrived at the second node may be transmitted out of the network or returned back to $Q_{2}$ for retransmission. The buffers of the nodes follow First-In First-Out (FIFO) technique for transmitting the packets through transmitters. After getting transmitted from the first transmitter the packets are forwarded to $Q_{2}$ for forward transmission with probability $(1-\theta)$ or returned back to the $Q_{1}$ with probability $\theta$. The packets arrived from the first transmitter are forwarded to $Q_{2}$ for transmission and exit from the network with probability (1- $\pi$ ) or returned back to the $Q_{2}$ with probability $\pi$. The service completion in both the transmitters follows Poisson processes with the parameters $\mu_{1}$ and $\mu_{2}$ for the first and second transmitters. The transmission rate of each packet is adjusted just before transmission depending on the content of the buffer connected to the transmitter. A schematic diagram representing the network model with two nodes and feedback for both nodes is shown in Fig. 1.

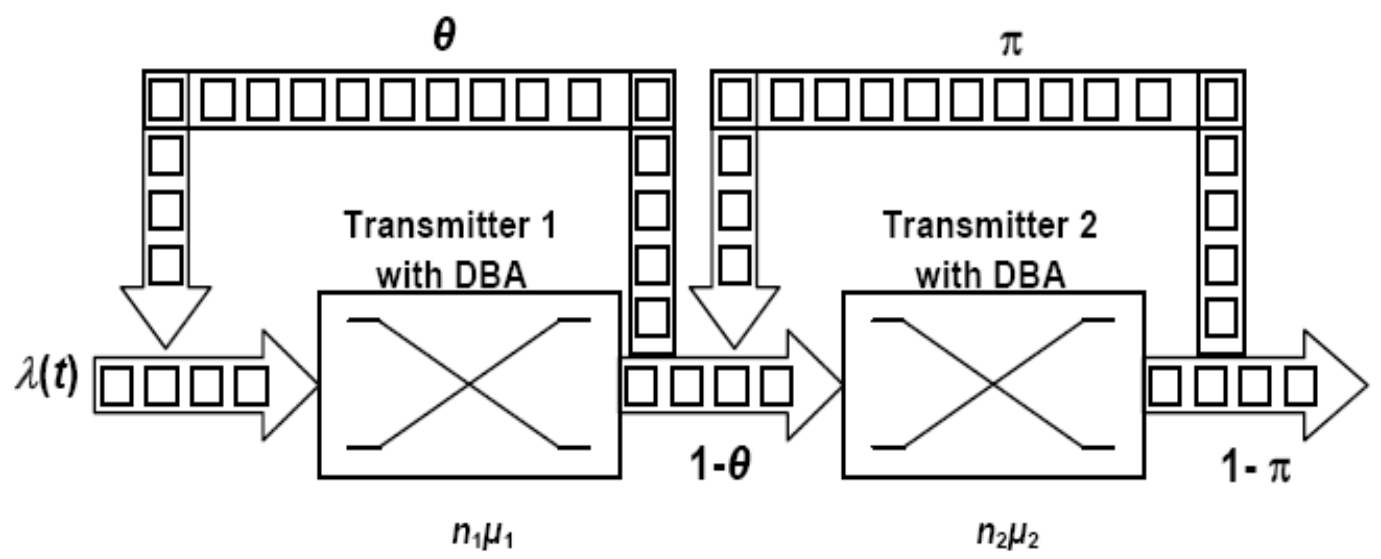

Fig. 1. Communication network model with two nodes.

Let $n_{1}$ and $n_{2}$ are the number of packets in first and second buffers and let $P_{n_{1}, n_{2}}{ }^{(t)}$ be the probability that there are $n_{1}$ packets in the first buffer and $n_{2}$ packets in the second buffer at time $t$. The differencedifferential equations for the above model are as follows: 


$$
\begin{gathered}
\frac{\partial P_{n_{1} n_{2}}(t)}{\partial t}=-\left(\lambda(t)+n_{1} \mu_{1}(1-\theta)+n_{2} \mu_{2}(1-\pi)\right) P_{n_{1}, n_{2}}(t)+\lambda(t) P_{n_{1}-1, n_{2}}(t)+\left(n_{1}+1\right) \mu_{1}(1-\theta) P_{n_{1}+1, n_{2}-1}(t)+\left(n_{2}+1\right) \mu_{2}(1-\pi) P_{n_{1}, n_{2}+1}(t) \\
\frac{\partial P_{n_{1}, 0}(t)}{\partial t}=-\left(\lambda(t)+n_{1} \mu_{1}(1-\theta)\right) P_{n_{1} 0}(t)+\lambda(t) P_{n_{1}-1,0}(t)+\mu_{2}(1-\pi) P_{n_{1}, 1}(t) \\
\frac{\partial P_{0, n_{2}}(t)}{\partial t}=-\left(\lambda(t)+n_{2} \mu_{2}(1-\pi)\right) P_{0, n_{2}}(t)+\mu_{1}(1-\theta) P_{n_{1}, n_{2}-1}(t)+\left(n_{2}+1\right) \mu_{2}(1-\pi) P_{0, n_{2}+1}(t) \\
\frac{\partial P_{0,0}(t)}{\partial t}=-\lambda(t) P_{0,0}(t)+\mu_{2}(1-\pi) P_{0,1}(t)
\end{gathered}
$$

Let $P\left(S_{1}, S_{2} ; t\right)$ be the joint probability generating function of $P_{n_{1}, n_{2}}{ }^{(t)}$. Then multiply the equation (1) with $S_{1}^{n_{1}} S_{2}^{n_{2}}$ and summing over all $n_{1}, n_{2}$ we get

$$
\begin{aligned}
\frac{\partial P\left(s_{1}, s_{2}: t\right)}{\partial t}= & -\sum_{n_{1}=1}^{\infty} \sum_{n_{2}=1}^{\infty} \lambda(t) P_{n_{1}, n_{2}}(t) s_{1}^{n_{1}} s_{2}^{n_{2}}-\sum_{n_{1}=1 n_{2}=1}^{\infty} n_{1} \mu_{1}(1-\theta) P_{n_{1}, n_{2}}(t) s_{1}^{n_{1}} s_{2}^{n_{2}}-\sum_{n_{1}=1}^{\infty} \sum_{n_{2}=1}^{\infty} n_{2} \mu_{2}(1-\pi) P_{n_{1}, n_{2}}(t) s_{1}^{n_{1}} s_{2}^{n_{2}}+ \\
& \lambda(t) \sum_{n_{1}=1}^{\infty} \sum_{n_{2}=1}^{\infty} P_{n_{1}-1, n_{2}}(t) s_{1}^{n_{1}} s_{2}^{n_{2}}+\sum_{n_{1}=1 n_{2}=1}^{\infty} \sum_{1}^{\infty}\left(n_{1}+1\right) \mu_{1}(1-\theta) P_{n_{1}+1, n_{2}-1}(t) s_{1}^{n_{1}} s_{2}^{n_{2}}+\sum_{n_{1}=1 n_{2}=1}^{\infty}\left(n_{2}+1\right) \mu_{2}(1-\pi) P_{n_{1}, n_{2}+1}(t)(t) s_{1}^{n_{1}} s_{2}^{n_{2}}- \\
& \sum_{n_{1}=1}^{\infty} \lambda(t) P_{n_{1}, 0}(t) s_{1}^{n_{1}}-\sum_{n_{1}=1}^{\infty} n_{1} \mu_{1}(1-\theta) P_{n_{1}, 0}(t) s_{1}^{n_{1}}+\sum_{n_{1}=1}^{\infty} \lambda(t) P_{n_{1}-1,0}(t) s_{1}^{n_{1}}+\sum_{n_{1}=1}^{\infty} \mu_{2}(1-\pi) P_{n_{1}, 1}(t) s_{1}^{n_{1}}-\sum_{n_{2}=1}^{\infty} \lambda(t) P_{0, n_{2}}(t) s_{2}^{n_{2}}- \\
& \sum_{n_{2}=1}^{\infty} n_{2} \mu_{2}(1-\pi) P_{0, n_{2}}(t) s_{2}^{n_{2}}+\mu_{1}(1-\theta) \sum_{n_{2}=1}^{\infty} P_{n_{1}, n_{2}-1}(t) s_{2}^{n_{2}}+\left(n_{2}+1\right) \mu_{2}(1-\pi) \sum_{n_{2}=1}^{\infty} P_{0, n_{2}+1}(t) s_{2}^{n_{2}}-\lambda(t) P_{0,0}(t)+\mu_{2}(1-\pi) P_{0,1}(t)
\end{aligned}
$$

After simplifying we get

$$
\begin{gathered}
\frac{\partial P\left(s_{1}, s_{2}: t\right)}{d t}=-\lambda(t) P\left(s_{1}, s_{2}: t\right)+\lambda(t) s_{1} P\left(s_{1}, s_{2}: t\right)-\mu_{1}(1-\theta) s_{1} \frac{\partial p}{\partial s_{1}}+ \\
\mu_{1}(1-\theta) . s_{2} \frac{\partial p}{\partial s_{1}}-\mu_{2}(1-\pi) s_{2} \frac{\partial p}{\partial s_{2}}+\mu_{2}(1-\pi) \frac{\partial p}{\partial s_{2}}
\end{gathered}
$$

Solving equation (3) by Lagrangian's method, we get the auxiliary equations as,

$$
\frac{d t}{1}=\frac{-d s_{1}}{\mu_{1}(1-\theta)\left(s_{2}-s_{1}\right)}=\frac{-d s_{2}}{\mu_{2}(1-\pi)\left(1-s_{2}\right)}=\frac{d p}{\lambda(t) P\left(s_{1}-1\right)}
$$

To solve the equations in (4) the functional form of $\lambda(t)$ is required. Let the mean arrival rate of packets is $\lambda(t)=\lambda+\alpha t$, where $\lambda>0, \alpha>0$ are constants.

Solving first and fourth terms in equation (4), we get

$$
a=\left(s_{2}-1\right) e^{\mu_{2}(1-\pi) t}
$$

Solving first and third terms in equation (4), we get

$$
b=\left(s_{1}-1\right) e^{-\mu_{1}(1-\theta) t}+\frac{\left(s_{2}-1\right) \mu_{1}(1-\theta) e^{-\mu_{1}(1-\theta) t}}{\left(\mu_{2}(1-\pi)-\mu_{1}(1-\theta)\right)}
$$

Solving first and second terms in equation (4), we get 


$$
c=P\left(S_{1}, S_{2} ; t\right) \exp \left\{-\left[\frac{\left(s_{1}-1\right)}{\mu_{1}(1-\theta)}\left[\lambda+\alpha t-\frac{\alpha}{\mu_{1}(1-\theta)}\right]+\frac{\left(s_{2}-1\right)}{\mu_{2}(1-\pi)}\left[\lambda+\alpha t-\left(\frac{\alpha\left(\mu_{1}(1-\theta)+\mu_{2}(1-\pi)\right)}{\mu_{1}(1-\theta) \cdot \mu_{2}(1-\pi)}\right)\right]\right]\right\}
$$

where $a, b$ and $c$ are arbitrary constants. The general solution of equation (4) gives the probability generating function of the number of packets in the first and second buffers at time $t$, as $P\left(S_{1}, S_{2} ; t\right)$.

$$
P=\exp \left\{\begin{array}{l}
\frac{S_{1}-1}{\mu_{1}(1-\theta)}\left(\lambda-\frac{\alpha}{\mu_{1}(1-\theta)}\right)\left(1-e^{-\mu_{1}(1-\theta) t}\right)+\frac{S_{1}-1}{\mu_{1}(1-\theta)} \alpha t+\frac{\left(S_{2}-1\right)\left(1-e^{-\mu_{2}(1-\pi) t}\right)}{\mu_{2}(1-\pi)}\left(\lambda-\frac{\alpha\left(\mu_{1}(1-\theta)+\mu_{2}(1-\pi)\right)}{\mu_{1}(1-\theta) \mu_{2}(1-\pi)}\right)+ \\
\frac{\left(S_{2}-1\right)}{\mu_{2}(1-\pi)} \alpha t+\frac{\left(S_{2}-1\right)}{\left(\mu_{2}(1-\pi)-\mu_{1}(1-\theta)\right)}\left(e^{\mu_{2}(1-\pi) t}-e^{\mu_{1}(1-\theta) t}\left(\lambda-\frac{\alpha}{\mu_{1}(1-\theta)}\right)\right.
\end{array}\right\}
$$

\section{Performance Measures of the Network Model}

In this section, we derive and analyze the performance measures of the network under transient conditions. Expand $P\left(S_{1}, S_{2} ; t\right)$ of equation (6) and collect the constant terms. From this, we get the probability that the network is empty as

$$
P_{00}(t)=\exp \left\{\begin{array}{l}
\frac{-1}{\mu_{1}(1-\theta)}\left(\lambda-\frac{\alpha}{\mu_{1}(1-\theta)}\right)\left(1-e^{\mu_{1}(1-\theta) t}\right)+\frac{-1}{\mu_{1}(1-\theta)} \alpha t+\frac{-1}{\mu_{2}(1-\pi)}\left(1-e^{-\mu_{2}(1-\pi) t}\right)\left(\lambda-\frac{\alpha\left(\mu_{1}(1-\theta)+\mu_{2}(1-\pi)\right)}{\mu_{1}(1-\theta) \mu_{2}(1-\pi)}\right)+ \\
\frac{-1}{\mu_{2}(1-\pi)} \alpha t+\frac{-1}{\left(\mu_{2}(1-\pi)-\mu_{1}(1-\theta)\right)}\left(e^{-\mu_{2}(1-\pi) t}-e^{-\mu_{1}(1-\theta) t}\right)\left(\lambda-\frac{\alpha}{\mu_{1}(1-\theta)}\right)
\end{array}\right\}
$$

Taking $S_{2}=1$ in equation (6) we get probability generating functions of the number of packets in the first buffer is

$$
P\left(\mathrm{~S}_{1} ; \mathrm{t}\right)=\exp \left\{\frac{S_{1}-1}{\mu_{1}(1-\theta)}\left(\lambda-\frac{\alpha}{\mu_{1}(1-\theta)}\right)\left(1-e^{-\mu_{1}(1-\theta) t}\right)+\frac{S_{1}-1}{\mu_{1}(1-\theta)} \alpha t\right\}
$$

Probability that the first buffer is empty as $\left(S_{1}=0\right)$

$$
P_{0 .}(t)=\exp \left\{\frac{-1}{\mu_{1}(1-\theta)}\left(\lambda-\frac{\alpha}{\mu_{1}(1-\theta)}\right)\left(1-e^{\mu_{1}(1-\theta) t}\right)+\frac{1}{\mu_{1}(1-\theta)} \alpha t\right\}
$$

Taking $S_{1}=1$ in equation (6) we get probability generating function of the number of packets in the first buffer is

$$
P\left(\mathrm{~S}_{2} ; \mathrm{t}\right)=\exp \left\{\begin{array}{l}
\frac{\left(S_{2}-1\right)\left(1-. e^{-\mu_{2}(1-\pi) t}\right)}{\mu_{2}(1-\pi)}\left(\lambda-\frac{\alpha\left(\mu_{1}(1-\theta)+\mu_{2}(1-\pi)\right)}{\mu_{1}(1-\theta) \mu_{2}(1-\pi)}\right)+\frac{\left(S_{2}-1\right)}{\mu_{2}(1-\pi)} \alpha t+ \\
\frac{\left(S_{2}-1\right)}{\left(\mu_{2}(1-\pi)-\mu_{1}(1-\theta)\right)}\left(e^{\mu_{2}(1-\pi) t}-e^{\mu_{1}(1-\theta) t}\right)\left(\lambda-\frac{\alpha}{\mu_{1}(1-\theta)}\right)
\end{array}\right\}
$$

Probability that the second buffer is empty as $\left(S_{2}=0\right)$ 


$$
P_{.0}(t)=\exp \left\{\begin{array}{l}
\frac{-1}{\mu_{2}(1-\pi)}\left(1-e^{-\mu_{2}(1-\pi) t}\right)\left(\lambda-\frac{\alpha\left(\mu_{1}(1-\theta)+\mu_{2}(1-\pi)\right)}{\mu_{1}(1-\theta) \mu_{2}(1-\pi)}\right)+\frac{-1}{\mu_{2}(1-\pi)} \alpha t+ \\
\frac{-1}{\left(\mu_{2}(1-\pi)-\mu_{1}(1-\theta)\right)}\left(e^{-\mu_{2}(1-\pi) t}-e^{-\mu_{1}(1-\theta) t}\right)\left(\lambda-\frac{\alpha}{\mu_{1}(1-\theta)}\right)
\end{array}\right\}
$$

Mean Number of Packets in the First Buffer is

$$
L_{1}(t)=\left\{\frac{1}{\mu_{1}(1-\theta)}\left(\lambda-\frac{\alpha}{\mu_{1}(1-\theta)}\right)\left(1-e^{\mu_{1}(1-\theta) t}\right)+\frac{-1}{\mu_{1}(1-\theta)} \alpha t\right\}
$$

Utilization of the first transmitter is

$$
U_{1}(t)=1-P_{0 .}(t)=1-\exp \left\{\frac{-1}{\mu_{1}(1-\theta)}\left(\lambda-\left(\frac{\alpha}{\mu_{1}(1-\theta)}\right)\left(1-e^{\mu_{1}(1-\theta) t}\right)+\frac{-1}{\mu_{1}(1-\theta)} \alpha t\right\}\right.
$$

Variance of the Number of packets in the first buffer is

$$
V_{1}(t)=\left\{\frac{1}{\mu_{1}(1-\theta)}\left(\lambda-\frac{\alpha}{\mu_{1}(1-\theta)}\right)\left(1-e^{\mu_{1}(1-\theta) t}\right)+\frac{-1}{\mu_{1}(1-\theta)} \alpha t\right\}
$$

Throughput of the first transmitter is

$$
T h_{1}=\mu_{1}\left(1-P_{0 .}(t)\right)=\mu_{1}\left(1+\exp \left\{\frac{-1}{\mu_{1}(1-\theta)}\left(\lambda-\left(\frac{\alpha}{\mu_{1}(1-\theta)}\right)\left(1-e^{\mu_{1}(1-\theta) t}\right)+\frac{1}{\mu_{1}(1-\theta)} \alpha t\right\}\right)\right.
$$

Average waiting time in the first buffer is

$$
W_{1}(t)=\frac{L_{1}(t)}{\mu_{1}\left(1-P_{0 .}(t)\right)}
$$

Mean number of packets in the second buffer is

$$
L_{2}(t)=\left\{\begin{array}{l}
\frac{1}{\mu_{2}(1-\pi)}\left(1-e^{-\mu_{2}(1-\pi) t}\right)\left(\lambda-\frac{\alpha\left(\mu_{1}(1-\theta)+\mu_{2}(1-\pi)\right)}{\mu_{1}(1-\theta) \mu_{2}(1-\pi)}\right)+\frac{1}{\mu_{2}(1-\pi)} \alpha t+ \\
\frac{1}{\left(\mu_{2}(1-\pi)-\mu_{1}(1-\theta)\right)}\left(e^{-\mu_{2}(1-\pi) t}-e^{-\mu_{1}(1-\theta) t}\right)\left(\lambda-\frac{\alpha}{\mu_{1}(1-\theta)}\right)
\end{array}\right\}
$$

Utilization of the second transmitter is

$$
U_{2}(t)=1-P_{.0}(t)=1-\exp \left\{\begin{array}{l}
\frac{-1}{\mu_{2}(1-\pi)}\left(1-e^{-\mu_{2}(1-\pi) t}\right)\left(\lambda-\frac{\alpha\left(\mu_{1}(1-\theta)+\mu_{2}(1-\pi)\right)}{\mu_{1}(1-\theta) \mu_{2}(1-\pi)}\right)+\frac{-1}{\mu_{2}(1-\pi)} \alpha t+ \\
\frac{-1}{\left(\mu_{2}(1-\pi)-\mu_{1}(1-\theta)\right)}\left(e^{-\mu_{2}(1-\pi) t}-e^{-\mu_{1}(1-\theta) t}\right)\left(\lambda-\frac{\alpha}{\mu_{1}(1-\theta)}\right)
\end{array}\right\}
$$

Variance of the number of packets in the second buffer is 


$$
V_{2}(t)=\left\{\begin{array}{l}
\frac{1}{\mu_{2}(1-\pi)}\left(1-e^{-\mu_{2}(1-\pi) t}\right)\left(\lambda-\frac{\alpha\left(\mu_{1}(1-\theta)+\mu_{2}(1-\pi)\right.}{\mu_{1}(1-\theta) \mu_{2}(1-\pi)}\right)+\frac{1}{\mu_{2}(1-\pi)} \alpha t+ \\
\frac{1}{\left(\mu_{2}(1-\pi)-\mu_{1}(1-\theta)\right)}\left(e^{-\mu_{2}(1-\pi) t}-e^{-\mu_{1}(1-\theta) t}\right)\left(\lambda-\frac{\alpha}{\mu_{1}(1-\theta)}\right)
\end{array}\right\}
$$

Throughput of the second transmitter is

$$
\begin{aligned}
\operatorname{Th}_{2}(t) & =\mu_{2}\left(1-P_{.0}(t)\right) \\
& =\mu_{2}\left(1+\exp \left\{\begin{array}{l}
\frac{1}{\mu_{2}(1-\pi)}\left(1-e^{-\mu_{2}(1-\pi) t}\right)\left(\lambda-\frac{\alpha\left(\mu_{1}(1-\theta)+\mu_{2}(1-\pi)\right)}{\mu_{1}(1-\theta) \mu_{2}(1-\pi)}\right)+\frac{1}{\mu_{2}(1-\pi)} \alpha t \\
+\frac{1}{\left(\mu_{2}(1-\pi)-\mu_{1}(1-\theta)\right)}\left(e^{-\mu_{2}(1-\pi) t}-e^{-\mu_{1}(1-\theta) t}\right)\left(\lambda-\frac{\alpha}{\mu_{1}(1-\theta)}\right)
\end{array}\right\}\right)
\end{aligned}
$$

Average waiting time in the second buffer is

$$
W_{2}(t)=\frac{L_{2}(t)}{\mu_{2}\left(1-P_{.0}(t)\right)}
$$

Mean number of packets in the entire network at time $t$ is

$$
L(t)=L_{1}(t)+L_{2}(t)
$$

Variability of the number of packets in the network is

$$
V(t)=V_{1}(t)+V_{2}(t)
$$

\section{Performance Evaluation of the Network Model}

In this section, the performance of the network model is discussed with numerical illustration. Different values of the parameters are taken for bandwidth allocation and arrival of packets. The packet arrival $(\lambda)$ varies from $2 \times 10^{4}$ packets/sec to $7 \times 10^{4}$ packets/sec, probability parameters $(\theta, \pi)$ varies from 0.1 to 0.9 , the transmission rate for first transmitter $\left(\mu_{1}\right)$ varies from $5 \times 10^{4}$ packets $/ \mathrm{sec}$ to $9 \times 10^{4}$ packets $/$ sec and transmission rate for second transmitter $\left(\mu_{2}\right)$ varies from $15 \times 10^{4}$ packets/sec to $19 \times 10^{4}$ packets $/$ sec. Dynamic Bandwidth Allocation strategy is considered for both the two transmitters. So, the transmission rate of each packet depends on the number of packets in the buffer connected to corresponding transmitter.

The equations (13), (15), (18) and (20) are used for computing the utilization of the transmitters and throughput of the transmitters for different values of parameters $t, \lambda, \alpha, \theta, \pi, \mu_{1}, \mu_{2}$ and the results are presented in the Table 1. The graphs in Fig. 2 show the relationship between utilization and throughput of the transmitters.

From the Table 1 it is observed that, when the time $(t)$ and $\lambda$ increases, the utilization of the transmitters is increasing for the fixed value of other parameters $\theta, \pi, \mu_{1}, \mu_{2}$. As the arrivals of the packets at the first node is non-homogeneous Poisson in nature, when the value of $\alpha$ increase from 0 to 2 , the throughput of the first and second transmitters is increasing for fixed values of other parameters. As the first transmitter probability parameter $\theta$ increases from 0.1 to 0.9 , the utilization of first transmitter increases and decreases for the second transmitter, due to the number of packets arriving at the second transmitter are decreasing as number of packets going back to the first transmitter in feedback are increasing. As the 
second transmitter probability parameter $\pi$ increases from 0.1 to 0.9 , the utilization of first transmitter remains constant and utilization of the second transmitter increases. This is because the number of packets arriving at the second transmitter is the packets arriving directly from the first transmitter and packets arrived for retransmission in feedback. As the transmission rate of the first transmitter $\left(\mu_{1}\right)$ increases from 5 to 9 , the utilization of the first transmitter decreases and the utilization of the second transmitter increases by keeping the other parameters as constant. As the transmission rate of the second transmitter $\left(\mu_{2}\right)$ increases from 15 to 19 , the utilization of the first transmitter is constant and the utilization of the second transmitter decreases by keeping the other parameters as constant.

Table 1. Values of Utilization and Throughput of the Network Model with DBA and Non-Homogeneous Poisson Arrivals

\begin{tabular}{|c|c|c|c|c|c|c|c|c|c|c|}
\hline$t$ & $\lambda$ & $\alpha$ & $\theta$ & $\pi$ & $\mu_{1}$ & $\mu_{2}$ & $U_{1}(t)$ & $U_{2}(t)$ & $T h_{1}(t)$ & $T h_{2}(t)$ \\
\hline 0.1 & 2 & 1 & 0.1 & 0.1 & 5 & 15 & 0.1524 & 0.0258 & 0.7621 & 0.3871 \\
\hline 0.3 & 2 & 1 & 0.1 & 0.1 & 5 & 15 & 0.3018 & 0.0938 & 1.5092 & 1.4065 \\
\hline 0.5 & 2 & 1 & 0.1 & 0.1 & 5 & 15 & 0.3716 & 0.1328 & 1.8579 & 1.9922 \\
\hline 0.7 & 2 & 1 & 0.1 & 0.1 & 5 & 15 & 0.4136 & 0.1560 & 2.0678 & 2.3399 \\
\hline 0.9 & 2 & 1 & 0.1 & 0.1 & 5 & 15 & 0.4447 & 0.1726 & 2.2233 & 2.5885 \\
\hline 0.5 & 3 & 1 & 0.1 & 0.1 & 5 & 15 & 0.4849 & 0.1853 & 2.4243 & 2.7792 \\
\hline 0.5 & 4 & 1 & 0.1 & 0.1 & 5 & 15 & 0.5777 & 0.2346 & 2.8887 & 3.5185 \\
\hline 0.5 & 5 & 1 & 0.1 & 0.1 & 5 & 15 & 0.6539 & 0.2809 & 3.2693 & 4.2131 \\
\hline 0.5 & 6 & 1 & 0.1 & 0.1 & 5 & 15 & 0.7163 & 0.3244 & 3.5814 & 4.8657 \\
\hline 0.5 & 7 & 1 & 0.1 & 0.1 & 5 & 15 & 0.7674 & 0.3653 & 3.8371 & 5.4788 \\
\hline 0.5 & 2 & 0 & 0.1 & 0.1 & 5 & 15 & 0.3281 & 0.1173 & 1.6403 & 1.7601 \\
\hline 0.5 & 2 & 0.5 & 0.1 & 0.1 & 5 & 15 & 0.3502 & 0.1251 & 1.7509 & 1.8766 \\
\hline 0.5 & 2 & 1 & 0.1 & 0.1 & 5 & 15 & 0.3716 & 0.1328 & 1.8579 & 1.9922 \\
\hline 0.5 & 2 & 1.5 & 0.1 & 0.1 & 5 & 15 & 0.3923 & 0.1404 & 1.9613 & 2.1067 \\
\hline 0.5 & 2 & 2 & 0.1 & 0.1 & 5 & 15 & 0.4123 & 0.1480 & 2.0613 & 2.2203 \\
\hline 0.5 & 2 & 1 & 0.1 & 0.1 & 5 & 15 & 0.3716 & 0.1328 & 1.8579 & 1.9922 \\
\hline 0.5 & 2 & 1 & 0.3 & 0.1 & 5 & 15 & 0.4216 & 0.1209 & 2.1081 & 1.8128 \\
\hline 0.5 & 2 & 1 & 0.5 & 0.1 & 5 & 15 & 0.4814 & 0.1028 & 2.4070 & 1.5414 \\
\hline 0.5 & 2 & 1 & 0.7 & 0.1 & 5 & 15 & 0.5517 & 0.0749 & 2.7586 & 1.1235 \\
\hline 0.5 & 2 & 1 & 0.9 & 0.1 & 5 & 15 & 0.6321 & 0.0311 & 3.1606 & 0.4660 \\
\hline 0.5 & 2 & 1 & 0.1 & 0.1 & 5 & 15 & 0.3716 & 0.1328 & 1.8579 & 1.9922 \\
\hline 0.5 & 2 & 1 & 0.1 & 0.3 & 5 & 15 & 0.3716 & 0.1625 & 1.8579 & 2.4376 \\
\hline 0.5 & 2 & 1 & 0.1 & 0.5 & 5 & 15 & 0.3716 & 0.2072 & 1.8579 & 3.1087 \\
\hline 0.5 & 2 & 1 & 0.1 & 0.7 & 5 & 15 & 0.3716 & 0.2861 & 1.8579 & 4.2916 \\
\hline 0.5 & 2 & 1 & 0.1 & 0.9 & 5 & 15 & 0.3716 & 0.3975 & 1.8579 & 5.9629 \\
\hline 0.5 & 2 & 1 & 0.1 & 0.1 & 5 & 15 & 0.3716 & 0.1328 & 1.8579 & 1.9922 \\
\hline 0.5 & 2 & 1 & 0.1 & 0.1 & 6 & 15 & 0.3337 & 0.1402 & 2.0025 & 2.1027 \\
\hline 0.5 & 2 & 1 & 0.1 & 0.1 & 7 & 15 & 0.3017 & 0.1454 & 2.1119 & 2.1811 \\
\hline 0.5 & 2 & 1 & 0.1 & 0.1 & 8 & 15 & 0.2745 & 0.1492 & 2.1959 & 2.2374 \\
\hline 0.5 & 2 & 1 & 0.1 & 0.1 & 9 & 15 & 0.2513 & 0.1519 & 2.2613 & 2.2784 \\
\hline 0.5 & 2 & 1 & 0.1 & 0.1 & 5 & 15 & 0.3716 & 0.1328 & 1.8579 & 1.9922 \\
\hline 0.5 & 2 & 1 & 0.1 & 0.1 & 5 & 16 & 0.3716 & 0.1258 & 1.8579 & 2.0132 \\
\hline 0.5 & 2 & 1 & 0.1 & 0.1 & 5 & 17 & 0.3716 & 0.1195 & 1.8579 & 2.0317 \\
\hline 0.5 & 2 & 1 & 0.1 & 0.1 & 5 & 18 & 0.3716 & 0.1138 & 1.8579 & 2.0481 \\
\hline 0.5 & 2 & 1 & 0.1 & 0.1 & 5 & 19 & 0.3716 & 0.1086 & 1.8579 & 2.0627 \\
\hline
\end{tabular}

It is also observed from the Table 1 that, as the time $(t)$ increases, the throughput of first and second transmitters is increasing for the fixed values of other parameters. When the parameter $\lambda$ increases from $3 \times 10^{4}$ packets/sec to $7 \times 10^{4}$ packets/sec, the throughput of both transmitters is increasing. As the parameter $\alpha$ increases, the throughput of both transmitters also increases. As the first probability 
parameter $\theta$ value increases from 0.1 to 0.9 , the throughput of the first transmitter increases and the throughput of the second transmitter decreases. As the second probability parameter $\pi$ value increases from 0.1 to 0.9 , the throughput of the first transmitter remains constant and the throughput of the second transmitter increases. As the transmission rate of the first transmitter $\left(\mu_{1}\right)$ increases from $5 \times 10^{4}$ packets/sec to $9 \times 10^{4}$ packets/sec, the throughput of the first and second transmitters is increasing. The transmission rate of the second transmitter $\left(\mu_{2}\right)$ increases from $15 \times 10^{4}$ packets/sec to $19 \times 10^{4}$ packets $/$ sec and the throughput of the first transmitter is constant and the throughput of the second transmitter is increasing.
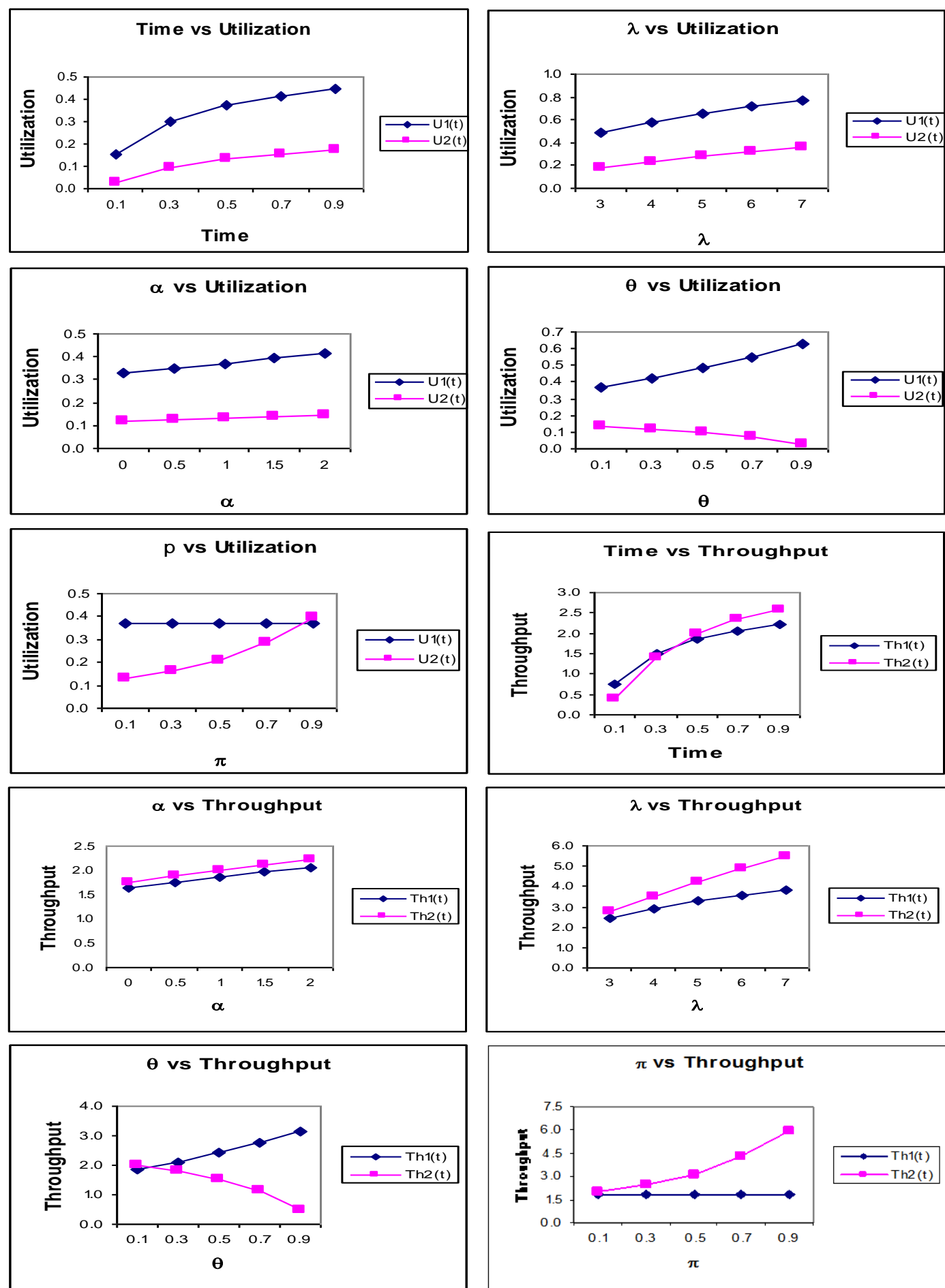

Fig. 2. The relationship between utilization and throughput and other parameters. 
Using equations (12), (17), (22) and (16), (21) the mean no. of packets in the two buffers and in the network, mean delay in transmission of the two transmitters are calculated for different values of $t, \lambda, \alpha, \theta$, $\pi, \mu_{1}, \mu_{2}$ and the results are shown in the Table 2 . The graphs showing the relationship between parameters and performance measure are shown in the Fig. 3.

Table 2. Values of Mean Number of Packets and Mean Delay of the Network Model with DBA and NonHomogeneous Arrivals

\begin{tabular}{|c|c|c|c|c|c|c|c|c|c|c|c|}
\hline$t$ & $\lambda$ & $\alpha$ & $\theta$ & $\pi$ & $\mu_{1}$ & $\mu_{2}$ & $L_{1}(t)$ & $L_{2}(t)$ & $L(t)$ & $W_{1}(t)$ & $W_{2}(t)$ \\
\hline 0.1 & 2 & 1 & 0.1 & 0.1 & 5 & 15 & 0.1654 & 0.0261 & 0.1915 & 0.2170 & 0.0675 \\
\hline 0.3 & 2 & 1 & 0.1 & 0.1 & 5 & 15 & 0.3593 & 0.0985 & 0.4578 & 0.2381 & 0.0700 \\
\hline 0.5 & 2 & 1 & 0.1 & 0.1 & 5 & 15 & 0.4645 & 0.1425 & 0.6070 & 0.2500 & 0.0715 \\
\hline 0.7 & 2 & 1 & 0.1 & 0.1 & 5 & 15 & 0.5337 & 0.1696 & 0.7033 & 0.2581 & 0.0725 \\
\hline 0.9 & 2 & 1 & 0.1 & 0.1 & 5 & 15 & 0.5882 & 0.1894 & 0.7776 & 0.2646 & 0.0732 \\
\hline 0.5 & 3 & 1 & 0.1 & 0.1 & 5 & 15 & 0.6633 & 0.2049 & 0.8682 & 0.2736 & 0.0737 \\
\hline 0.5 & 4 & 1 & 0.1 & 0.1 & 5 & 15 & 0.8621 & 0.2673 & 1.1294 & 0.2985 & 0.0760 \\
\hline 0.5 & 5 & 1 & 0.1 & 0.1 & 5 & 15 & 1.0609 & 0.3297 & 1.3907 & 0.3245 & 0.0783 \\
\hline 0.5 & 6 & 1 & 0.1 & 0.1 & 5 & 15 & 1.2597 & 0.3921 & 1.6519 & 0.3517 & 0.0806 \\
\hline 0.5 & 7 & 1 & 0.1 & 0.1 & 5 & 15 & 1.4585 & 0.4545 & 1.9131 & 0.3801 & 0.0830 \\
\hline 0.5 & 2 & 0 & 0.1 & 0.1 & 5 & 15 & 0.3976 & 0.1248 & 0.5224 & 0.2424 & 0.0709 \\
\hline 0.5 & 2 & 0.5 & 0.1 & 0.1 & 5 & 15 & 0.4311 & 0.1337 & 0.5647 & 0.2462 & 0.0712 \\
\hline 0.5 & 2 & 1 & 0.1 & 0.1 & 5 & 15 & 0.4645 & 0.1425 & 0.6070 & 0.2500 & 0.0715 \\
\hline 0.5 & 2 & 1.5 & 0.1 & 0.1 & 5 & 15 & 0.4980 & 0.1513 & 0.6493 & 0.2539 & 0.0718 \\
\hline 0.5 & 2 & 2 & 0.1 & 0.1 & 5 & 15 & 0.5315 & 0.1602 & 0.6917 & 0.2578 & 0.0721 \\
\hline 0.5 & 2 & 1 & 0.1 & 0.1 & 5 & 15 & 0.4645 & 0.1425 & 0.6070 & 0.2500 & 0.0715 \\
\hline 0.5 & 2 & 1 & 0.3 & 0.1 & 5 & 15 & 0.5475 & 0.1288 & 0.6763 & 0.2597 & 0.0711 \\
\hline 0.5 & 2 & 1 & 0.5 & 0.1 & 5 & 15 & 0.6566 & 0.1084 & 0.7651 & 0.2728 & 0.0703 \\
\hline 0.5 & 2 & 1 & 0.7 & 0.1 & 5 & 15 & 0.8023 & 0.0779 & 0.8802 & 0.2908 & 0.0693 \\
\hline 0.5 & 2 & 1 & 0.9 & 0.1 & 5 & 15 & 1.0000 & 0.0316 & 1.0316 & 0.3164 & 0.0677 \\
\hline 0.5 & 2 & 1 & 0.1 & 0.1 & 5 & 15 & 0.4645 & 0.1425 & 0.6070 & 0.2500 & 0.0715 \\
\hline 0.5 & 2 & 1 & 0.1 & 0.3 & 5 & 15 & 0.4645 & 0.1773 & 0.6419 & 0.2500 & 0.0728 \\
\hline 0.5 & 2 & 1 & 0.1 & 0.5 & 5 & 15 & 0.4645 & 0.2322 & 0.6968 & 0.2500 & 0.0747 \\
\hline 0.5 & 2 & 1 & 0.1 & 0.7 & 5 & 15 & 0.4645 & 0.3370 & 0.8016 & 0.2500 & 0.0785 \\
\hline 0.5 & 2 & 1 & 0.1 & 0.9 & 5 & 15 & 0.4645 & 0.5067 & 0.9712 & 0.2500 & 0.0850 \\
\hline 0.5 & 2 & 1 & 0.1 & 0.1 & 5 & 15 & 0.4645 & 0.1425 & 0.6070 & 0.2500 & 0.0715 \\
\hline 0.5 & 2 & 1 & 0.1 & 0.1 & 6 & 15 & 0.4061 & 0.1510 & 0.5571 & 0.2028 & 0.0718 \\
\hline 0.5 & 2 & 1 & 0.1 & 0.1 & 7 & 15 & 0.3591 & 0.1571 & 0.5162 & 0.1700 & 0.0720 \\
\hline 0.5 & 2 & 1 & 0.1 & 0.1 & 8 & 15 & 0.3209 & 0.1615 & 0.4824 & 0.1461 & 0.0722 \\
\hline 0.5 & 2 & 1 & 0.1 & 0.1 & 9 & 15 & 0.2894 & 0.1647 & 0.4541 & 0.1280 & 0.0723 \\
\hline 0.5 & 2 & 1 & 0.1 & 0.1 & 5 & 15 & 0.4645 & 0.1425 & 0.6070 & 0.2500 & 0.0715 \\
\hline 0.5 & 2 & 1 & 0.1 & 0.1 & 5 & 16 & 0.4645 & 0.1345 & 0.5990 & 0.2500 & 0.0668 \\
\hline 0.5 & 2 & 1 & 0.1 & 0.1 & 5 & 17 & 0.4645 & 0.1273 & 0.5918 & 0.2500 & 0.0626 \\
\hline 0.5 & 2 & 1 & 0.1 & 0.1 & 5 & 18 & 0.4645 & 0.1208 & 0.5853 & 0.2500 & 0.0590 \\
\hline 0.5 & 2 & 1 & 0.1 & 0.1 & 5 & 19 & 0.4645 & 0.1149 & 0.5795 & 0.2500 & 0.0557 \\
\hline
\end{tabular}

It is observed from the Table 2 that as the time $(t)$ varies from 0.1 to 0.9 seconds, the mean number of packets in the two buffers and in the network are increasing when other parameters are kept constant. When the $\lambda$ changes from $3 \times 10^{4}$ packets/second to $7 \times 10^{4}$ packets/second the mean number of packets in the first, second buffers and in the network are increasing. As the parameter $\alpha$ increases from 0 to 2 the mean number of packets in both transmitters is increasing. As the first probability parameter $\theta$ varies from 0.1 to 0.9 , the mean number packets in the first buffer increases and decreases in the second buffer due to feedback for the first buffer. When the second probability parameter $\pi$ varies from 0.1 to 0.9 , the mean 
number packets in the first buffer remains constant and increases in the second buffer due to packets arrived directly from the first transmitter and packets for retransmission due to feedback from the second transmitter. When the transmission rate of the first transmitter $\left(\mu_{1}\right)$ varies from $5 \times 10^{4}$ packets/second to $9 \times 10^{4}$ packets/second, the mean number of packets in the first buffer decreases, in the second buffer increases and in the network decreases. When the transmission rate of the second transmitter $\left(\mu_{2}\right)$ varies from $15 \times 10^{4}$ packets/second to $19 \times 10^{4}$ packets/second, the mean number of packets in the first buffer remains constant and decreases in the second buffer and in the network.
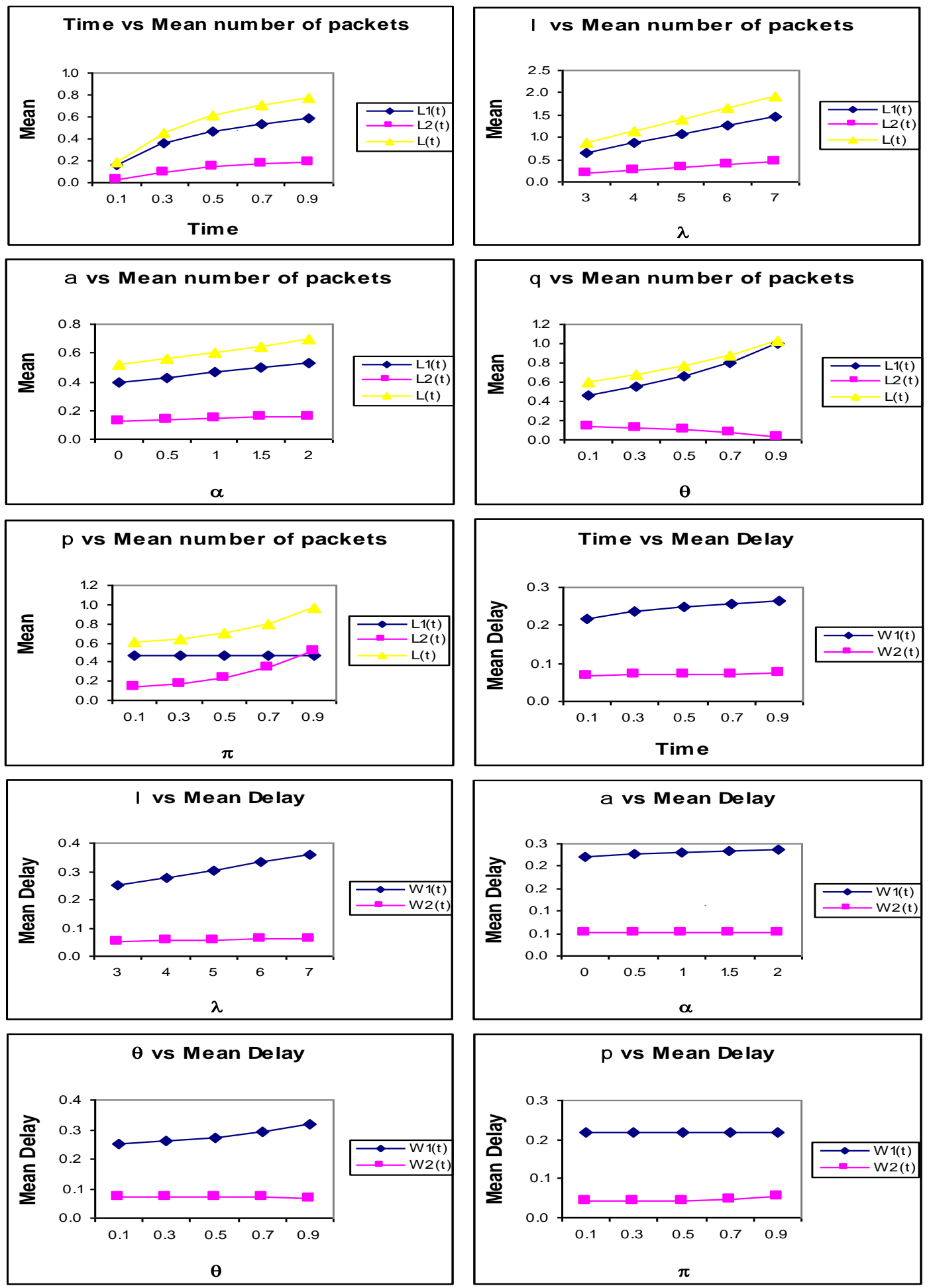

Fig. 3. The relationship between mean no. of packets, mean delay and various parameters. 
From the Table 2, it is also observed that with time $(t)$ and $\lambda$, the mean delay in the two buffers are increasing for fixed values of other parameters. When the parameter $\alpha$ increases from 0 to 2 , the mean delay in the two buffers also increases. As the parameter $\theta$ varies the mean delay in the first buffer increases and decreases in the second buffer due to feedback for the first buffer. As the parameter $\pi$ varies the mean delay in the first buffer remains constant and increases in the second buffer. As the transmission rate of the first transmitter $\left(\mu_{1}\right)$ varies, the mean delay of the first buffer decreases, in the second buffer slightly increases. When the transmission rate of the second transmitter $\left(\mu_{2}\right)$ varies, the mean delay of the first buffer remains constant and decreases for the second buffer.

From the above analysis, it is observed that the dynamic bandwidth allocation strategy has a significant influence on all performance measures of the network. We also observed that the performance measures are highly sensitive towards smaller values of time. Hence, it is optimal to consider dynamic bandwidth allocation and evaluate the performance under transient conditions. It is also to be observed that the congestion in buffers and delays in transmission can be reduced to a minimum level by adopting dynamic bandwidth allocation.

\section{Conclusion}

This paper introduces a tandem communication network model with two transmitters with dynamic bandwidth allocation and feedback for both transmitters. Arrival of packets at the two buffers follows non homogeneous Poisson arrivals and dynamic bandwidth allocation at the transmitters. The dynamic bandwidth allocation is adapted by immediate adjustment of packet service time by utilizing idle bandwidth in the transmitter. The transient analysis of the model is capable of capturing the changes in the performance measures of the network like average content of the buffers, mean delays, throughput of the transmitters, idleness of the transmitters etc, explicitly. It is observed that the feedback probability parameters $(\theta, \pi)$ have significant influence on the overall performance of the network. The numerical study reveals that the proposed communication network model is capable of evaluating and predicting the performance of communication networks more close to the reality.

\section{References}

[1] Balsamo, S., Persone, V. D. N., \& Onvural, R. (2001). Analysis of Queuing Networks with Blocking. Boston: Kluwer Academic Publishers.

[2] Clo, M. C. (1998). MVA for product-form cyclic queuing networks with blocking. Annals of Operations Research, 79, 83-96.

[3] Martin, J. B. (2002). Large tandem queuing networks with blocking. Queuing Systems, 41, 45-72.

[4] Onvural, R. Survey of closed queuing networks with blocking. Computer Survey, 22, 83-121.

[5] Perros, H.G. (1994). Queuing Networks with Blocking. Exact and Approximate Solution. New York: Oxford University Press.

[6] Tang, J., \& Zhao, Y. Q. (2008). Stationary tail asymptotic of a tandem queue with feedback. Annals of Operation Research, 160, 173-189.

[7] Gun, L., \& Makowski, A. M. (1989). Matrix-geometric solution for two node tandem queueing systems with phase-type servers subject to blocking and failures. Stochastic Models, 5, 431-457.

[8] Gun, L., \& Makowski, A. M. (1987). Matrix-geometric solution for finite capacity queues with phase-type distributions. In P.-J. Courtois, \& G. Latouche (Eds.), Performance'87 (pp. 269-282). North-Holland.

[9] Mei, R. D. V. D., et al. (2002). Response times in a two-node queueing network with feedback. Performance Evaluation, 49, 99-110.

[10] Gnedenko, B. W., \& Konig, D. (1983). Handbuch der Bedienungstheorie. Berlin: Akademie Verlag. 
[11] Gaujal, B., \& Hyon, E., (2002). Optimal routing policies in deterministic queues in tandem. Proceedings of Sixth International Workshop on Discrete Event Systems (pp. 251-257).

[12] Parthasarathy, P. R., \& Selvraju, N. (2001). Transient analysis of a queue where potential customers are discouraged by queue length. Mathematical Problems in Engineering, 7, 433-454.

[13] Srinivasa R. K., Varma P. S., \& Srinivas, Y. (2008). Interdependent queuing model with start-up delay. Journal of Statistical Theory and Applications, 7(2), 219.

[14] Sriram, K. (1993). Methodologies for bandwidth allocation, transmission scheduling and congestion avoidance in broadband ATM networks, 43-59.

[15] Padmavathi, G., Rao K. S., \& Reddy, K. V. V. S. (2009). Performance evaluation of parallel and series communication network with dynamic bandwidth allocation. CIIT International Journal of Networking and Communication Engineering, 1(7), 410-421.

[16] Varma P. S., \& Rao, K. S. (2007). A Communication network with load dependent transmission. International Journal of Mathematical Sciences, 6(2), 199-210.

[17] Rao, K. N., Rao, K. S., \& Rao, P. S. (2010). A tandem Communication network with DBA and modified phase type Transmission having bulk arrivals. International Journal of Computer Science, 7(3), 18-26.

[18] Sundari, M. V. R., Rao, K. S., Rao, P. S., \& Varma, P. S. (September 2011). On tandem communication network model with DBA having NHP arrivals for first node and Poisson process arrivals for second node. International Journal of Computer Science, 8(2), 136-144.

[19] Sundari, M. V. R., Rao, K. S., Rao, P. S., \& Varma, P. S. (October 2011). Three node tandem communication network model with dynamic bandwidth allocation and Non Homogeneous Poisson arrivals. International Journal of Computer Applications, 31(1).

[20] Raghavendran, C. V., Satish, G. N., Sundari, M. V. R., Varma, P. S. (July 2014). Transient analysis of communication network model with homogeneous Poisson arrivals and dynamic bandwidth allocation. International Journal of Computer Applications, 98(3).

[21] Raghavendran, C. V., Satish, G. N., Sundari, M. V. R., Varma, P. S. (July 2014). Tandem communication network model with DBA having Non Homogeneous Poisson arrivals and feedback for first node. International Journal of Computers and Technology, 13(9).

[22] Raghavendran, C. V., Satish, G. N., Sundari, M. V. R., Varma, P. S. (2014). Performance analysis of a two node tandem communication network with feedback. Global Journal of Computer Science and Technology (G), 14(1).

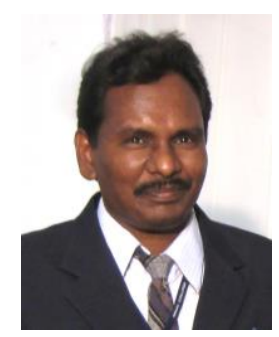

Ch V. Raghavendran is a research scholar in Computer Science Department of Adikavi Nannaya University, Rajahmundry, AP, India. His is working as an associate professor in Ideal College, Kakinada, AP, India. He has presented several papers in various national and international conferences. His areas of interest are communication networks, mobile ad hoc networks, swarm intelligence and data mining.

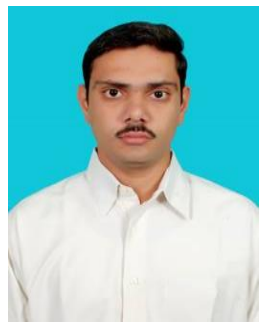

G. Naga Satish is a research scholar in Computer Science Department of Adikavi Nannaya University, Rajahmundry, AP. At present he is working as an associate professor in Ideal College, Kakinada, AP, India. He has presented and published papers in national and international conferences. His areas of interest include computer networks and network security. 


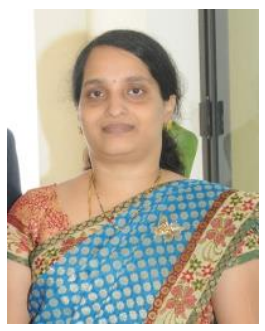

M. V. Rama Sundari is working as an associate professor in Department of IT, Godavari Institute of Engineering and Technology, Rajahmundry, AP, India. She published several research papers in national and international journals. Her areas of interest include cloud computing and networks.

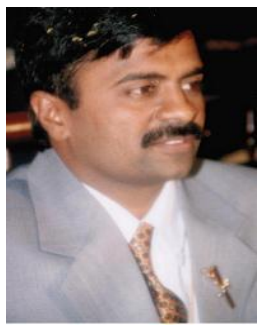

P. Suresh Varma is a professor in the Department of Computer Science, Adikavi Nannaya University, Rajahmundry, India. He has published several research papers in national and international journals and presented papers at various conferences, seminars and workshops. His current research interests are communication networks, data mining and cloud computing. 